Pacific Journal of Mathematics

TOPOLOGICAL GROUPS WHICH SATISFY AN OPEN 


\title{
TOPOLOGICAL GROUPS WHICH SATISFY AN OPEN MAPPING THEOREM
}

\author{
Douglass L. Grant
}

Let $\mathscr{C}$ be a category of Hausdorff topological groups. A Hausdorff topological group $G$ is called a $B(\mathscr{C})$ group if every continuous and almost open homomorphism from $G$ onto a group in $\mathscr{C}$ is open. An internal characterization of such groups is obtained. For certain $\mathscr{C}$, the permanence properties of $B(\mathscr{C})$ groups and related categories are investigated, with some positive results pertaining to products and subobjects, and several counterexamples. Forms of the closed graph theorem for topological groups are then obtained which generalize results of T. Husain.

1. Definitions and permanence properties. Given a topological group $G$ with topology $u$, we shall denote the filter of neighbourhoods of the identity by $\mathscr{V}(G)$ or $\mathscr{V}(u)$, and closures by $\mathrm{Cl}_{G}$ or $\mathrm{Cl}_{u}$, depending on the emphasis desired. If $u$ and $v$ are two group topologies on a group $G$, then $v(u)$ will denote that group topology on $G$ having as a fundamental system of unit neighbourhoods the collection $\left\{\mathrm{Cl}_{v} U: U \in \mathscr{V}(u)\right\}$. The set of closed normal subgroups of a topological group $G$ will be denoted by $\mathcal{N}(G)$. A homomorphism $f: G \rightarrow H$ of topological groups is said to be almost open (resp., almost continuous) if the image (resp., inverse image) of a unit neighbourhood is dense in a unit neighbourhood. An isomorphism of topological groups is a group isomorphism which is both continuous and open.

Let $\mathscr{C}$ be a category of Hausdorff topological groups. After [8], we say that a Hausdorff group $G$ is a $B(\mathscr{C})$ group if every continuous and almost open homomorphism from $G$ onto a group in $\mathscr{C}$ is open, and that $G$ is a $B_{r}(\mathscr{C})$ group if every homomorphism with these properties which is also one-to-one is open. We reserve the symbol $\mathscr{A}$ for the category of all Hausdorff topological groups.

Husain [8] showed that locally compact groups and complete metrizable groups are $B(\mathscr{A})$ groups, while Brown [2, Theorem 4] showed that any topological group complete in the sense of Čech has the $B(\mathscr{A})$ property. A minimal topological group (i.e., one with its coarsest compatible Hausdorff topology) is easily seen to be a $B_{r}(\mathscr{A})$ group. Other examples will be mentioned later.

Husain also observed [8, Theorem 31.4] that a topological group $(G, u)$ is a $B_{r}(\mathscr{A})$ group iff, for every Hausdorff group topology $v$ on $G$ such that $v \subseteq u$ and $v(u)=v$, it follows that $u=v$. We give analogous 
statements for $B_{r}(\mathscr{C})$ and $B(\mathscr{C})$ groups, where $\mathscr{C}$ satisfies a very mild condition. For a topological group $(G, u)$ and $H \in \mathcal{N}(G)$, let $u H$ denote the group topology on $G$ having the collection $\{U H: U \in \mathscr{V}(G)\}$ as a subbasis of unit neighbourhoods, and $u / H$ the quotient topology on $G / H$. We require a lemma which follows directly from Proposition 30.3 of $[8]$.

LemMA 1.1. A topological group $G$ is a $B(\mathscr{C})$ group iff $G / H$ is a $B_{r}(\mathscr{C})$ group for every $H \in \mathcal{N}(G)$.

The following definition is adapted from Isbell [10, p. 119]. Let $\mathscr{X}$ be a category, $\mathscr{Y}$ a subcategory of $\mathscr{X}, \mathscr{F}$ a class of morphisms in $\mathscr{X}$. Then $\mathscr{Y}$ is said to be right fitting with respect to $\mathscr{F}$ if $X \in \mathscr{X}, Y \in \mathscr{Y}, f: Y \rightarrow X$ a morphism in $\mathscr{F}$ together imply $X \in \mathscr{Y}$. Let $\mathscr{H}$ denote the class of isomorphisms of Hausdorff topological groups. (More extensive use of this notion, involving other classes of maps, will be made in §2.)

THEOREM 1.2. Let $\mathscr{C}$ be a subcategory of $\mathscr{A}$ which is right fitting with respect to $\mathscr{H}$.

(a) A topological group $(G, u)$ is a $B_{r}(\mathscr{C})$ group iff, for every group topology $v$ on $G$ such that $(G, v) \in \mathscr{C}, v \subseteq u$, and $v(u)=v$, it follows that $v=u$.

(b) A topological group $(G, u)$ is a $B(\mathscr{C})$ group iff, for every $H \in \mathcal{N}(G)$ and every group topology $v$ on $G$ such that $(G / H, v / H) \in \mathscr{C}$, $v \subseteq u H$, and $v(u H)=v$, it follows that $v=u H$.

Proof. Part (a) follows in a manner similar to Theorem 31.4 of [8]. One then obtains (b) by invoking Lemma 1.1, applying (a) to the quotient groups, and observing that every group topology on a group $G / H$ coarser than the quotient topology arises from a group topology on $G$ coarser than $u H$.

Investigation of some permanence properties of $B(\mathscr{A})$ and $B_{r}(\mathscr{A})$ groups was carried out by L. J. Sulley [15], who gave criteria for the inheritance of these properties by dense subgroups and by completions, in the Abelian case. His assumption of commutativity can be removed quite painlessly, however. The proof of the next lemma proceeds in a fashion nearly identical to that of the corresponding result in [15].

LEMMA 1.3. Let $E$ be a Hausdorff group, $G$ a dense subgroup of $E$, $H \in \mathcal{N}(E), q: E \rightarrow E / H$ the natural map. Then the map $r: G \rightarrow q(G)$ obtained by restricting $q$ is continuous and almost open. Furthermore, $r$ is open iff $H \cap G$ is dense in $H$.

THEOREM 1.4. Let $G$ be a Hausdorff group, $E$ its completion with respect to its two-sided uniformity. 
(a) $G$ is a $B(\mathscr{A})$ group iff $E$ is a $B(\mathscr{A})$ group and $G \cap H$ is dense in $H$ for every $H \in \mathcal{N}(E)$.

(b) $G$ is a $B_{r}(\mathscr{A})$ group iff $E$ is a $B_{r}(\mathscr{A})$ group and $G \cap H$ is nontrivial for every nontrivial $H \in \mathcal{N}(E)$.

Proof. The "only if" parts of (a) and (b) follow as in [15], using Lemma 1.3. For the "if" part of (a), let $F$ be any Hausdorff group, $F^{\prime}$ its completion with respect to its two-sided uniformity, $f$ a continuous, almost open homomorphism of $G$ onto $F$. By Proposition 5, p. 246 of [1], $f$ has a unique extension $f^{\prime}: E \rightarrow F^{\prime}$, which can be shown to be almost open onto its range. The balance follows as in [15], using Lemma 1.3. The proof of the "if" part of (b) is similar, with the additional observation that the extension $f^{\prime}$ of the one-to-one homomorphism $f$ is also one-to-one.

It follows from this criterion $[15,16]$ that the group $U$ of complex roots of unity is a $B(\mathscr{A})$ group, while, for instance, neither the group $Q$ of rationals nor the group $U_{p}$ of $p$-power roots of unity is a $B_{r}(\mathscr{A})$ group.

Clearly, if a product of groups has the $B(\mathscr{A})$ property, then each factor has this property. Using Theorem 1.4, however, we can show that neither the class of $B(\mathscr{A})$ groups nor that of $B_{r}(\mathscr{A})$ groups is closed even under finite Cartesian products.

EXAMPLE 1. Let $R$ denote the reals with usual topology and $T$ the circle group, and let $U$ be as above. All of these groups are $B(\mathscr{A})$ groups, but $R \times U$ is not even a $B_{r}(\mathscr{A})$ group. The Hausdorff completion of this group is $R \times T$, which is locally compact and so a $B(\mathscr{A})$ group. Let $\beta$ represent any irrational number. Then $R \times T$ has a non-trivial subgroup

$$
H=\{(n, \exp 2 n \pi \beta i): n \in Z\},
$$

which is discrete and therefore closed, and whose intersection with $R \times U$ is trivial. It follows from Theorem 1.4(b) that $R \times U$ is not a $B_{r}(\mathscr{A})$ group, and perforce not a $B(\mathscr{A})$ group. The same argument, applied to the product of $U$ with the discrete group of integers, shows that this product also fails to have the $B_{r}(\mathscr{A})$ property.

The following example shows that certain special products retain the property, however.

EXAMPLE 2. We show that any finite power of $U$ is a $B(\mathscr{A})$ group. Soundararajan [13] has called a subgroup $H$ of a topological group $G$ totally dense if $H \cap L$ is dense in $L$ for every closed subgroup $L$ of $G$. For Abelian groups $G$, this coincides with the property described in Theorem 1.4(a). Letting $\langle x\rangle$ denote the subgroup generated by an 
element $x \in G$, he asserts that $H$ is totally dense in $G$ iff $H \cap \mathrm{Cl}_{G}\langle x\rangle$ is dense in $\mathrm{Cl}_{G}\langle x\rangle$ for all $x \in G$. Since the completion $T^{n}$ of $U^{n}$ is compact and so a $B(\mathscr{A})$ group, it is therefore sufficient to show that

$$
\mathrm{Cl}_{T^{n}}\left(U^{n} \cap \mathrm{Cl}_{T^{n}}\langle x\rangle\right)=\mathrm{Cl}_{T^{n}}\langle x\rangle
$$

for all $x=\left(x_{1}, x_{2}, \cdots, x_{n}\right) \in T^{n}$.

We may assume without loss of generality that for some nonnegative integers $r, s$, the entries $x_{1}, \cdots, x_{r}$ are elements of $U$, that $x_{r+1}, \cdots, x_{r+s}$ are images under the exponential map of irrational numbers $\alpha_{1}, \cdots, \alpha_{s}$ which, together with 1 , form a linearly independent set over $Q$, and that the balance of the $x_{t}$ are images of linear combinations over $Q$ of the $\alpha_{j}$ and 1. By the linear independence of $\left\{1, \alpha_{1}, \cdots, \alpha_{s}\right\}$, it follows from Theorem 443 of [5] that $H=\mathrm{Cl}_{T^{n}}\langle x\rangle=F \times T^{s} \times C(M)$, where $F$ is a finite subgroup of $T^{r}$ (and hence of $U^{r}$ ), $M$ is an $(s+1) \times(n-r-s$ ) matrix with rational entries, and

$$
C(M)=\left\{(\exp )^{n-r-s}\left(k\left(\alpha_{1}, \cdots, \alpha_{s}, 1\right) M\right): k \in Z\right\} .
$$

It can be seen that the intersection of $H$ with $U^{n}$ is dense in $H$, whence $(\Delta)$ is satisfied. Hence, $U^{n}$ is a $B(\mathscr{A})$ group for any positive integer $n$.

If $G_{1}$ is totally dense in $G$ and $G_{1} \subseteq G_{2} \subseteq G$, then $G_{2}$ is totally dense in $G$. It then follows that $U^{n} \times T^{m}$ is totally dense in $T^{n+m}$ for any positive integers $n$ and $m$, and so is a $B(\mathscr{A})$ group. Since Stephenson [14, Theorem 2] has shown that totally dense subgroups of compact groups are minimal, it also follows that $U^{n}$ is a minimal topological group. Since the product of a minimal group with a compact group is minimal [14], if further follows that $U^{n} \times K$ is a minimal topological group, and so a $B_{r}(\mathscr{A})$ group, for any compact group $K$.

As to subobjects, it does not appear to be true, in general, that our two properties are inherited by closed or even closed normal subgroups. However, some partial results of a positive nature have been obtained. A subgroup $H$ of a topological group $G$ is said to be a retract of $G$ if there is a continuous homomorphism $r: G \rightarrow H$ whose restriction to $H$ is the identity. By [6, p. 59] and [17, pp. 20 and 95], $H$ is normal and a retract of $G$ iff there exists a subgroup $H^{\prime}$ of $G$ such that the multiplication map $m: H \times H^{\prime} \rightarrow G$ is an isomorphism.

PROPOSITION 1.5. Let $H$ be a normal subgroup of $G$ and a retract thereof. If $G$ is a $B(\mathscr{A})$ (resp., $B_{r}(\mathscr{A})$ ) group, then $H$ is a $B(\mathscr{A})$ (resp., $\left.B_{r}(\mathscr{A})\right)$ group.

Proof. The case for $B(\mathscr{A})$ groups follows at once, since the projection map $H \times H^{\prime} \rightarrow H$ is continuous and open. Now, let $G$ be a 
$B_{r}(\mathscr{A})$ group, $A$ any Hausdorff group, $m$ as above, $j$ the continuous inverse of $m$, and $f: H \rightarrow A$ a continuous, almost open, bijective homomorphism. Then $h=j\left(f \times i d_{H^{\prime}}\right)$ is also bijective, and, since $f$ is almost open and $G$ is a $B_{r}(\mathscr{A})$ group, it follows that $h$ is open. Hence, $m h=f \times i d_{H^{\prime}}$ is open, whence $f$ is open.

The following two lemmas are required to establish the next permanence property, and will also be used extensively in $\$ 2$.

DEFINITION. If $f: G \rightarrow H$ is a homomorphism (not necessarily continuous) of topological groups, let $\mathscr{V}_{f}=\left\{V^{*}=\right.$ $\left.V f\left[\mathrm{Cl}_{G} f^{-1}(V)\right]: V \in \mathscr{V}(H)\right\}$.

LEMMA 1.6. Let $f:(G, u) \rightarrow(H, v)$ be a homomorphism. If $\mathscr{V}_{f}$ is a subbasis for the unit neighbourhood filter of a group topology $v_{f}$ and the graph of $f$ is closed in $G \times H$, then $\left(H, v_{f}\right)$ is a Hausdorff topological group.

Husain defined a topology $w$ related to $v_{f}$ in [9], and established a similar result concerning it. The proof of Lemma 1.6 follows in a manner parallel to his, with certain simplifications arising from the elimination of one closure operator from the definition of the sets in the unit neighbourhood basis.

Definition. For a subgroup $K$ of a group $G$, let Cent $_{G} K$ denote the centralizer of $K$ in $G$, and $S_{G}(K)=K$ Cent $_{G} K$.

LEMMA 1.7. (a) If $f(G)$ is dense in $H$, then $\mathscr{V}_{f}$ is a subbasis for the unit neighbourhood filter of a group topology $v_{f}$, and $\mathscr{W}_{f}=$ $\left\{\mathrm{Cl}_{H} f\left[\mathrm{Cl}_{G} f^{-1}(V)\right]=\hat{V}: V \in \mathscr{V}(H)\right\}$ is also a subbasis of unit neighbourhoods for $v_{f}$.

(b) If $K$ is an open subgroup of a topological group $(G, u)$ such that $S_{G}(K)$ is dense in $G$ and $w$ is a group topology on $K$, then $\mathscr{V}(w)$ is also a fundamental system of unit neighbourhoods for a group topology on $G$.

Proof. (a) Clearly $\hat{V} \subseteq V^{*}$, for every $V \in \mathscr{V}(H)$. Now $f\left[f^{-1}(V)\right]=V \cap f(G)$, which is dense in $V$, and so $V \subseteq \hat{V}$. Now, if $V_{1}^{2} \subseteq V$, then

$$
V_{1}^{*}=V_{1} f\left[\mathrm{Cl}_{G} f^{-1}\left(V_{1}\right)\right] \subseteq V_{1} \hat{V}_{1} \subseteq\left(\hat{V}_{1}\right)^{2} \subseteq \hat{V}
$$

Thus, $\mathscr{W}_{f}$ generates the same filter as $\mathscr{V}_{f}$.

To show that $v_{f}$ is a group topology, we show that $\mathscr{W}_{f}$ satisfies $\left(G V_{\mathrm{I}}^{\prime}\right)-\left(G V_{\mathrm{III}}^{\prime}\right)$ of [1, p. 222-3]. The first two follow immediately, since $(\hat{V})^{2} \subseteq\left(V^{2}\right)^{\wedge}$ and $\left(V^{-1}\right)^{\wedge}=(\hat{V})^{-1}$. As for $\left(G V_{\text {III }}^{\prime}\right)$, let $V, V_{1} \in \mathscr{V}(H)$, $a \in H, t \in G$ such that $V_{1}=V_{1}^{-1}, V_{1}^{3} \subseteq V$, and $a \in V_{1} f(t)$. Then 


$$
\begin{aligned}
& a\left[f(t)^{-1} V_{1} f(t)\right]^{\wedge} a^{-1} \subseteq V_{1} f(t) \cdot f(t)^{-1} V_{1} f(t) \cdot f(t)^{-1} V_{1} \\
& \quad=V_{1} \hat{V}_{1} V_{1} \subseteq\left(\hat{V}_{1}\right)^{3} \subseteq\left(V_{1}^{3}\right)^{\wedge} \subseteq \hat{V} .
\end{aligned}
$$

(b) It is necessary only to show $\mathscr{V}(w)$ satisfies $\left(G V_{\text {III }}^{\prime}\right)$. Let $U, U_{1} \in \mathscr{V}(G), x \in G, k \in K, c \in \operatorname{Cent}_{G} K$ such that $U_{1}=U_{1}^{-1}, U_{1}^{3} \subseteq U$ and $x \in U_{1} c k$. Then

$$
x\left(k^{-1} U_{1} k\right) x^{-1} \subseteq U_{1} c k\left(k^{-1} U_{1} k\right) k^{-1} c^{-1} U_{1}=U_{1}\left(c U_{1} c^{-1}\right) U_{1}=U_{1}^{3} \subseteq U .
$$

PROPOSITION 1.8. Let $\mathscr{C}$ be a category of Hausdorff groups such that every $B_{r}(\mathscr{C})$ group is in $\mathscr{C}$. Then every closed central subgroup of a $B_{r}(\mathscr{C})$ (resp., $B(\mathscr{C})$ ) group is a $B_{r}(\mathscr{C})$ (resp., $B(\mathscr{C})$ ) group.

Proof. Let $(G, u)$ be a $B_{r}(\mathscr{C})$ group, $K$ a closed central subgroup of $G, w$ any group topology on $K$ such that $(K, w) \in \mathscr{C}, w \subseteq u \mid K$ and $w(u \mid K)=w$. Since $K$ is central in $G$, it is routine to show that $\mathscr{V}(v)=\{U W: U \in \mathscr{V}(u), W \in \mathscr{V}(w)\}$ is a subbasis for the unit neighbourhood filter of a group topology $v$ on $G$. The graph of the natural injection $K \rightarrow G$ is closed in $(K, w) \times(K, w)$, and so in $(K, w) \times(G, u)$, since $K$ is $u$-closed. From this and the fact that $w(u \mid K)=w$, it follows from Lemma 1.6 that $v$ is Hausdorff. By Proposition 31.8 of [8], one has $v \subseteq v(u)$. However, if $U, U_{1} \in \mathscr{V}(u)$ such that $U_{1}^{2} \subseteq U$, then $\mathrm{Cl}_{v} U \subseteq$ $\left(\mathrm{Cl}_{v} U_{1}\right)^{2} \subseteq U_{1} \mathrm{Cl}_{w}\left(U_{1} \cap K\right) \in \mathscr{V}(v)$. Hence, $v(u)=v$, and the identity map $(G, u) \rightarrow(G, v)$ is continuous and almost open. Then $(G, v)$ is a $B_{r}(\mathscr{C})$ group, and so is in $\mathscr{C}$. Hence, $v=u$, whence $u|K=v| K=$ $w$. Therefore, $K$ is a $B_{r}(\mathscr{C})$ group.

The case for $B(\mathscr{C})$ groups is proved in a similar fashion, letting $H \in \mathcal{N}(K), w$ a topology on $K$ such that $(K / H, w / H) \in \mathscr{C}, w \subseteq(u \mid K) H$ and $w[(u \mid K) H]=w$. One can then define $v_{H}$ by $\mathscr{V}\left(v_{H}\right)=$ $\{U W: U \in \mathscr{V}(H), W \in \mathscr{V}(w)\}$ and show that $v_{H}=u H$, whence $u H \mid K=$ $v_{H} \mid K=w$, and $(K, u \mid K)$ is a $B(\mathscr{C})$ group.

Proposition 1.9. Let $\mathscr{C}$ be as in Proposition $1.8,(G, u)$ a $B_{r}(\mathscr{C})$ group (resp., $B(\mathscr{C})$ group) with equal left and right uniform structures. Then any closed subgroup $K$ of $G$ such that $S_{G}(K)$ is dense in $G$ is a $B_{r}(\mathscr{C})$ (resp., $B(\mathscr{C})$ ) group.

Proof. Let the topologies $u \mid K, w$ and $v$ be as in Proposition 1.8. Without loss of generality, we may assume that an element of $\mathscr{V}(u)$ is fixed under all inner automorphisms of $G$ [7, p. 22]. For such a neighbourhood $U$ and any $A \subseteq G$, we then have $A U=U A$. It is then easy to see that $\mathscr{V}(v)$ satisfies $\left(G V_{\mathrm{I}}^{\prime}\right)$ and $\left(G V_{\mathrm{II}}^{\prime}\right)$ of $[1, \mathrm{p} .222-3]$. To see that $\left(G V_{\mathrm{III}}^{\prime}\right)$ is also satisfied, let $x \in G$, and $U W, U_{1} W_{1} \in \mathscr{V}(v)$ such that $U_{1}, W_{1}$ are symmetric and $\left(U_{1} W_{1}\right)^{3} \subseteq U W$. Then there exist elements 
$a, b$ of $K$ and of its centralizer, respectively, such that $x \in$ $U_{1} W_{1} a b$. Then

$$
\begin{aligned}
& x U_{1}\left(a^{-1} W_{1} a\right) x \subseteq U_{1} W_{1} a b U_{1}\left(a^{-1} W_{1} a\right)\left(b^{-1} a^{-1} U_{1} W_{1}\right) \\
& =U_{1} W_{1} U_{1} b W_{1} b^{-1} U_{1} W_{1}=\left(U_{1} W_{1}\right)^{3} \subseteq U W .
\end{aligned}
$$

As in Proposition 1.8, it follows that $v$ is Hausdorff and that $v(u)=v$, whence $(G, v)$ is a $B_{r}(\mathscr{C})$ group and so is in $\mathscr{C}$. Then $v=u$, and so $u|K=v| K=w$. Hence, $(K, u \mid K)$ is a $B_{r}(\mathscr{C})$ group.

Now let $(G, u)$ be a $B(\mathscr{C})$ group, then choose $H$ and $w$ and construct $v_{H}$ as in the analogous case of Proposition 1.8. Since $(G, u)$ has equal uniformities and $H$ is normal in $K$, for $U W \in \mathscr{V}\left(v_{H}\right), U_{1} U_{1}^{-1} \subseteq U$, $W_{1} W_{1}^{-1} \subseteq W$, we have $\left(U_{1} W_{1}\right)\left(U_{1} W_{1}\right)^{-1} \subseteq U W$. The continuity of the conjugation maps follows in a manner similar to the $B_{r}(\mathscr{C})$ case. It then follows that $v_{H}=u H$, as in Proposition 1.8.

Remarks. (i) A closed subgroup $K$ of $G$ such that $S_{G}(K)$ is dense in $G$ is necessarily normal in $G$, since $S_{G}(K)$ is a subgroup of the normalizer of $K$ in $G$, and the normalizer is closed [4].

(ii) The condition that $G$ have equal uniformities can be replaced by the slightly weaker condition that $G$ has a fundamental system of unit neighbourhoods fixed under all conjugations by elements of $K$.

(iii) Clearly, $\mathscr{A}$ satisfies the condition imposed on $\mathscr{C}$ in Propositions 1.8 and 1.9. Indeed, this condition is satisfied by any category right fitting with respect to isomorphisms, if one were to modify the definition of $B(\mathscr{C})$ (resp., $B_{r}(\mathscr{C})$ ) groups to require the existence of at least one continuous, almost open (resp., and one-to-one) homomorphism onto a group in $\mathscr{C}$, thus precluding a vacuous satisfaction of the definition from [8].

Let $\mathscr{E}$ denote the class of morphisms in $\mathscr{A}$ which are almost open.

Proposition 1.10 . Let $\mathscr{C}$ be such that either (i) every $B_{r}(\mathscr{C})$ group is in $\mathscr{C}$, or (ii) $\mathscr{C}$ is right fitting with respect to $\mathscr{E}$. Then any open subgroup $K$ of a $B_{r}(\mathscr{C})$ (resp., $B(\mathscr{C})$ ) group $G$ such that $S_{G}(K)$ is dense in $G$ is a $B_{r}(\mathscr{C})$ (resp., $B(\mathscr{C})$ ) group.

Proof. Under either condition, let $w$ be a group topology on $K$ such that $(K, w) \in \mathscr{C}, w \subseteq u \mid K$ and $w(u \mid K)=w$. Let $v$ be the topology on $G$ having as its unit neighbourhood filter $\mathscr{V}(v)=$ $\{U W: U \in \mathscr{V}(u), W \in \mathscr{V}(w)\}$. Let $j:(K, w) \rightarrow(G, u)$ be the natural injection. By our assumptions on $w$, it follows that $v$ induces the $v_{j}$ topology on $K$, and, by Lemma 1.7 (a) and (b), $\mathscr{V}(v)$ generates a group topology on $G$. As in Proposition 1.8, we have $v \subseteq u$ and $v(u)=v$.

Now, if $\mathscr{C}$ satisfies (i), we observe that the identity map 
$(G, u) \rightarrow(G, v)$ is continuous and almost open, whence $(G, v)$ is a $B_{r}(\mathscr{C})$ group and so in $\mathscr{C}$. If $\mathscr{C}$ satisfies (ii), we observe that the natural injection $(K, w) \rightarrow(G, v)$ is continuous and almost open, so $(G, v) \in \mathscr{C}$.

It then follows that $v=u$, since $(G, u)$ is a $B_{r}(\mathscr{C})$ group, and so $u|K=v| K=w$. Therefore, $(K, u \mid K)$ is a $B_{r}(\mathscr{C})$ group.

The analogous statement for the $B(\mathscr{C})$ case is proved by first letting $H \in \mathcal{N}(K)$ and $w$ be a group topology such that $(K / H, w / H) \in \mathscr{C}$, $w \subseteq(u \mid K) H$ and $w[(u \mid K) H]=w$, and proceeding as above.

To demonstrate some more perverse properties of these categories, we now display counterexamples, concerned with join topołogies, direct limits and quotients.

EXAMPle 3. Let $(R, u)$ denote the reals with the usual topology, $g$ a discontinuous automorphism of the reals, and $(R, g(u))$ the reals endowed with the topology consisting of images under $g$ of $u$-open sets. Then $g$ is a homeomorphism from $(R, u)$ to $(R, g(u))$, whence $(R, g(u))$ is locally compact and so a $B(\mathscr{A})$ group.

The identity map $j:(R, u \vee g(u)) \rightarrow(R, u)$ is clearly continuous, and is also almost open, since the image under $g$ of any $u$-open set is $u$-dense in $R$ [7, p. 49]. However, $j$ is plainly not open. Hence, $(R, u \vee g(u))$ is not even a $B_{r}(\mathscr{A})$ group. This example also shows, of course, that the join of two locally compact group topologies is not necessarily locally compact. Thanks are due to E. Dubinsky for suggesting the above example in the latter context.

ExAmple 4. Let $(R, d)$ denote the reals with discrete topology, $(R, u)$ as in Example 3 . Let $G_{1}=(R, u) \times(R, d), G_{2}=(R, d) \times(R, u)$, and let $f: G_{1} \rightarrow G_{2}$ be defined by $(x, y) \mapsto(y, x)$, and let this system be ordered by $1<2$. Its inductive limit in the category of topological spaces is then $R^{2}$ endowed with the topology $(u \times d) \wedge(d \times u)$. It is proved in [12], however, that this is not even a group topology, although the groups involved are locally compact and hence $B(\mathscr{A})$ groups.

ExAmple 5. Let $T$ be as in Example 1, $G$ the subgroup of $T$ consisting of those elements of squarefree order. It is shown in [15] that $G$ is a $B_{r}(\mathscr{A})$ group which is not a $B(\mathscr{A})$ group. It then follows from Lemma 1.1 that not all quotients of $G$ can be $B_{r}(\mathscr{A})$ groups. This counterexample shows that the $B_{r}(\mathscr{A})$ property is not divisible, and thus that the portion of Proposition 31.7 of [8] which refers to $B_{r}(\mathscr{C})$ groups is false. Gaps are thereby created in the proofs of Theorems 32.8 and 32.9 of [8]. A corrected version of the former appears in $\$ 2$.

A sixth example, which follows, shows that, for the class $\mathscr{C}_{1}$ of first countable Hausdorff groups, the $B(\mathscr{A})$ groups form a proper subclass of the $B\left(\mathscr{C}_{1}\right)$ groups. We first observe that, since a countably compact 
subspace of a first countable space is closed [3, p. 230], it follows that a continuous, almost open homomorphism of a locally countably compact group into a first countable group is open. Therefore, every locally countably compact group, and hence every countably compact Hausdorff group, is a $B\left(\mathscr{C}_{1}\right)$ group.

EXAMPLE 6. Let $S$ be any uncountable set, and let $G$ be any compact Hausdorff group with nontrivial centre. Let $B=G^{s}$, and define

$$
P=\left\{\left(x_{\alpha}\right): x_{\alpha} \neq e \text { for at most countably many } \alpha \in S\right\} .
$$

By $[11$, p. 127], $P$ is countably compact and a proper dense subgroup of the group $B$, which is compact and so a $B_{r}(\mathscr{A})$ group. For each $g \in G$, let $(g)$ denote the element $\left(x_{\alpha}\right)$ of $B$ such that $x_{\alpha}=g$ for all $\alpha \in S$. It is easy to see that $H=\{(g): g \in$ Cent $G\}$ is a nontrivial closed normal subgroup of $B$, and that $H \cap P=\{(e)\}$. By Theorem 1.4(b), it follows that $P$ is not a $B_{r}(\mathscr{A})$, and perforce not a $B(\mathscr{A})$, group.

2. Closed graph theorems. In [8], Husain announced a quite general form of the closed graph theorem for topological groups (Theorem 32.5), and drew an extensive list of corollaries therefrom. However, the proof of this theorem contained a serious flaw, acknowledged by Husain in [9], where he salvaged some of the results from [8]. In this section, we salvage more results from [8] by weakening the assumption of commutativity of the codomain imposed by Husain in [9].

Let us recall the definition of "right fitting" from $\S 1$, and agree to denote the graph of a mapping $f$ by $R(f)$ throughout the balance of the paper.

THEOREM 2.1. Let $\mathscr{C}$ be a category of Hausdorff groups which is right fitting with respect to $\mathscr{E}$. Let $(G, u) \in \mathscr{C},(H, v)$ be a $B_{r}(\mathscr{C})$ group, $f: G \rightarrow H$ an almost continuous, almost open homomorphism with closed graph such that $f(G)$ is dense in $H$. Then $f$ is continuous.

Proof. By Lemmas 1.7(a) and 1.6, $v_{f}$ is a Hausdorff group topology. Since $v_{f} \subseteq v$, the identity map $j:(H, v) \rightarrow\left(H, v_{f}\right)$ is continuous. By Proposition 31.8 of [8], we have that $v_{f} \subseteq v_{f}(v) \subseteq$ $v$. Letting $U, V \in \mathscr{V}(H)$ such that $V^{2} \subseteq U$, we proceed as in Theorem 32.5 of [8] to show that $V^{*} \subseteq \mathrm{Cl}_{v_{f}} U$, and conclude that $v_{f}(v)=v_{f}$, whence $j$ is almost open. Then $g=j f$ is almost open, and also continuous, since $g^{-1}(U) \supseteq g^{-1}\left(j f\left[\mathrm{Cl}_{G} f^{-1}(U)\right]\right) \supseteq \mathrm{Cl}_{G} f^{-1}(U)$, which is in $\mathcal{V}(G)$. Thus, $g \in$ 
$\mathscr{E},\left(H, v_{f}\right) \in \mathscr{C}$, and $j$ is open, since $(H, v)$ is a $B_{r}(\mathscr{C})$ group. Hence, $v_{f}=v, g=f$, and $f$ is continuous.

Corollary 2.2. Let $\mathscr{C}$ be as in Theorem 2.1. Let $(G, u) \in \mathscr{C}$, $(H, v)$ be a $B_{r}(\mathscr{C})$ group, $f: G \rightarrow H$ an almost continuous, almost open homomorphism with closed graph such that $S_{H}\left[\mathrm{Cl}_{H} f(G)\right]$ is dense in $H$. Then $f$ is continuous.

Proof. By Proposition $1.10, K=\mathrm{Cl}_{H} f(G)$ is a $B_{r}(\mathscr{C})$ group, and it follows that the corestriction of $f$ to $K$ is continuous, by Theorem 2.1. Hence, $f$ is continuous.

THEOREM 2.3. Let $\mathscr{C}$ be any category of Hausdorff groups such that every $B_{r}(\mathscr{C})$ group is in $\mathscr{C}$. Let $(G, u) \in \mathscr{C},(H, v)$ be a $B_{r}(\mathscr{C})$ group and $f: G \rightarrow H$ an almost continuous, almost open homomorphism with closed graph such that $f(G)$ is dense in $H$. Then $f$ is continuous.

Proof. This proof parallels that of Theorem 2.1, except that the fact that $\left(H, v_{f}\right)$ is in $\mathscr{C}$ is deduced by observing that $j:(H, v) \rightarrow\left(H, v_{f}\right)$ continuous and almost open implies $\left(H, v_{f}\right)$ is a $B_{r}(\mathscr{C})$ group.

The next corollary follows in a manner similar to Corollary 2.2.

Corollary 2.4. Let $\mathscr{C}$ be as in Theorem 2.3. Let $(G, u) \in \mathscr{C}$, $(H, v)$ be a $B_{r}(\mathscr{C})$ group, $f: G \rightarrow H$ an almost continuous, almost open homomorphism with closed graph such that $S_{H}\left[\mathrm{Cl}_{H} f(G)\right]$ is dense in $H$. Then $f$ is continuous.

For categories $\mathscr{C}$ of groups which satisfy the condition of Theorem 2.3 , we can remove the "almost open" hypothesis on the map at the cost of adding certain other hypotheses. A preliminary lemma is required.

LEMMA 2.5. If either (i) $f(G) \subseteq$ Cent $H$, or (ii) $H$ has equal uniformities and $S_{H}[f(G)]$ is dense in $H$, then $v_{f}$ is a group topology.

Proof. The proof in case (i) is obvious. For (ii), we once again use $\left(G V_{\mathrm{I}}^{\prime}\right)-\left(G V_{\text {III }}^{\prime}\right)$ of $[1$, p. 222-3], obtaining the first two in a manner parallel to that of Proposition 1.8. To obtain $\left(G V_{\text {III }}^{\prime}\right)$, let $V^{*} \in \mathscr{V}_{f}$, and select $W^{*} \in \mathscr{V}_{f}$ such that $W$ is symmetric and invariant under conjugations, and $\left(W^{*}\right)^{3} \subseteq V^{*}$. Let $x \in H, t \in G$ and $b \in \operatorname{Cent}_{H}[f(G)]$ such that $x \in f(t) b W^{*}$. Then

$$
x W^{*} x^{-1} \subseteq f(t) b\left(W^{*}\right)^{3} b^{-1} f(t)^{-1}=\left[f(t) W^{*} f(t)^{-1}\right]^{3} .
$$


But $f(t) f\left[\mathrm{Cl}_{G} f^{-1}(W)\right] f(t)^{-1}=f\left(\mathrm{Cl}_{G}\left[f^{-1}\left(f(t) W f(t)^{-1}\right)\right]\right)=f\left[\mathrm{Cl}_{G} f^{-1}(W)\right]$. By virtue of this and the invariance of $W$ under conjugations, it follows that

$$
x W^{*} x^{-1} \subseteq\left[f(t) W^{*} f(t)^{-1}\right]^{3}=\left(W^{*}\right)^{3} \subseteq V^{*}
$$

THEOREM 2.6. Let $\mathscr{C}$ be as in Theorem 2.3. Let $(G, u) \in \mathscr{C},(H, v)$ be a $B_{r}(\mathscr{C})$ group $f: G \rightarrow H$ an almost continuous homomorphism with closed graph such that either (i) $f(G) \subseteq$ Cent $H$, or (ii) $H$ has equal uniformities and $S_{H}[f(G)]$ is dense in $H$. Then $f$ is continuous.

Proof. By Lemma 2.5, $v_{f}$ is a group topology in either case. As in Theorem 2.1, it follows that $v_{f}(v)=v$, and as in Theorem 2.3, $\left(H, v_{f}\right)$ is a $B_{r}(\mathscr{C})$ group and so in $\mathscr{C}$. Thus, $v_{f}=v$, and $f$ is continuous.

As with previous results, we point out that Theorem 2.6 holds, in particular, for $\mathscr{C}=\mathscr{A}$. The conditions on the homomorphism can be further relaxed if additional topological conditions are imposed on the groups involved.

Definition. A group $G$ is called weakly separable [2] if, for every $V \in \mathscr{V}(G)$, there exists a countable subset $X_{V}$ of $G$ such that $V X_{V}=$ $G$. (This property clearly generalizes both separability and the Lindelöf property.)

The proof of the next lemma parallels that of Proposition 32.11(b) of [8], which this result generalizes.

LEMMA 2.7. Any homomorphism from a Hausdorff group with the Baire property to a weakly separable group is almost continuous.

THEOREM 2.8. Let $\mathscr{C}$ be as in Theorem 2.3. Let $G$ be a group in $\mathscr{C}$ with the Baire property, $H$ a weakly separable $B_{r}(\mathscr{C})$ group. Then a homomorphism $f: G \rightarrow H$ with closed graph is continuous if either (i) $f(G) \subseteq$ Cent $H$, or (ii) $H$ has equal uniformities and $S_{H}\left[\mathrm{Cl}_{H} f(G)\right]$ is dense in $H$.

Proof. By Lemma 2.7, $f$ is almost continuous. Then $f$ is continuous by Theorem 2.6.

These considerations also allow us to prove a form of the open mapping theorem which corrects and extends Theorem 32.8 of [8].

THEOREM 2.9. Let $\mathscr{C}$ be as in Theorem 2.3. Let $G$ be a $B(\mathscr{C})$ group with equal uniformities, $H$ any Hausdorff group. Then any almost open homomorphism $g$ of $G$ onto $H$ with closed graph is open. 
Proof. Let $K$ be the kernel of $g$ and $n: G \rightarrow G / K$ the quotient map. Let $g=f n$. Since $R(g)$ is closed and contains $K \times\left\{e_{H}\right\}$, which is normal in $G \times H$, it follows by Corollary 24.4 of [8] that $R(f)$ is closed.

By Proposition 30.3 of [8], $f$ is almost open, whence $f^{-1}$ is almost continuous, $f^{-1}$ also has closed graph, clearly. Now $G / K$ is a $B(\mathscr{C})$ group, by Proposition 31.7 of [8], so $f^{-1}$ is continuous, by Theorem 2.6. Hence, $f$ is open and so is $g$, by Proposition 30.3 of [8].

Finally, let $\mathscr{D}$ denote the class of morphisms in $\mathscr{A}$ which have image dense in the codomain.

THEOREM 2.10. Let $\mathscr{C}$ be a category of Hausdorff groups which is right fitting with respect to $\mathscr{D}, G \in \mathscr{C},(H, v)$ a $B_{r}(\mathscr{C})$ group. Then an almost continuous homomorphism $f: G \rightarrow(H, v)$ with closed graph is in $\mathscr{D}$ if $f(G)$ is dense in $H$.

Proof. As before, we form the $v_{f}$ topology and observe, by Lemmas 1.6 and $1.7(\mathrm{a})$ that $v_{f}$ is a Hausdorff group topology. Letting $j:(H, v) \rightarrow\left(H, v_{f}\right)$ be the identity map, we further observe that $g=j f$ is continuous, as in Theorem 2.1. Since $v_{f} \subseteq v, g(G)$ is dense in $\left(H, v_{f}\right)$, so $g \in \mathscr{D}$, and $\left(H, v_{f}\right) \in \mathscr{C}$. Also as in Theorem 2.1, it follows that $j$ is continuous and almost open, whence $j$ is open, since $(H, v)$ is a $B_{r}(\mathscr{C})$ group. Therefore, $v_{f}=v, f$ is continuous, and $f \in \mathscr{D}$.

In closing, we note that the "right fitting" properties mentioned above are by no means exotic. Among the categories of Hausdorff groups which are right fitting with respect to $\mathscr{D}$ are the compact, precompact, Abelian, connected and separable groups, and among those right fitting with respect to $\mathscr{E}$ are the locally compact, locally precompact, metrizable and locally connected groups. Groups with equal uniformities, second countable groups and Abelian profinite groups are right fitting with respect to $\mathscr{E} \cap \mathscr{D}$.

Portions of this paper were included in the author's Ph.D. thesis, written at McMaster University under the direction of Dr. Taqdir Husain. His guidance was greatly appreciated, as were the most helpful comments and suggestions of the referee. The author also gratefully acknowledges the support of the government of the Province of Ontario while he was a graduate student, and, subsequently, of the National Research Council of Canada and of the Council for Research of St. Francis Xavier University.

\section{REFERENCES}

1. N. Bourbaki, Elements of Mathematics, General Topology, Part 1, Addison-Wesley, Reading, 1966. 
2. L. G. Brown, Topologically complete groups, Proc. Amer. Math. Soc., 35 (1972), 593.

3. J. Dugundji, Topology, Allyn and Bacon, Boston, 1966.

4. D. L. Grant, Centralizers and normalizers in Hausdorff groups, Math. Mag., 48 (1975), 218.

5. G. H. Hardy and E. M. Wright, The Theory of Numbers, 3rd Edition, Oxford, 1954.

6. E. Hewitt and K. A. Ross, Abstract Harmonic Analysis, Springer-Verlag, 1963.

7. E. Hewitt and K. Stromberg, Real and Abstract Analysis, Spring-Verlag, 1965.

8. T. Husain, Introduction to Topological Groups, Saunders, Philadelphia, 1966.

9. _ - On a closed graph theorem for topological groups, Proc. Japan Acad., 44 (1968), 446.

10. J. R. Isbell, Uniform Spaces, Amer. Math. Soc. Colloquium Publications, Providence, 1964.

11. L. Pontryagin, Topological Groups, Princeton University Press, Princeton, 1946.

12. P. Samuel, Ultrafilters and compactifications of uniform spaces, Trans. Amer. Math. Soc., 64 (1948), 100.

13. T. Soundararajan, Totally dense subgroups of topological groups, in general topology and its applications to modern analysis and algebra, Proceedings of the Kanpur Topological Conference, 1968, Academia, Prague, 1968.

14. R. M. Stephenson, Jr., Minimal topological groups, Math. Ann., 192 (1971), 193.

15. L. J. Sulley, A note on B- and Br-complete topological Abelian groups, Proc. Camb. Phil. Soc., 66 (1969), 275.

16. C. Sunyach, Compléments au théorème du graphe fermé, C. R. Acad. Sci. Paris, 267 (1968), 215.

17. A. Weil, L'integration dans les groupes topologiques et ses applications, Actualités Sci. et Ind.

869, 1145, Hermann et Cie., Paris, 1941 and 1951.

Received June 24, 1974 and in revised form January 7, 1977.

College of Cape Breton,

Sydney, Nova Scotia, CANada 



\section{PACIFIC JOURNAL OF MATHEMATICS}

\section{EDITORS}

RichaRd ARENS (Managing Editor)

University of California

Los Angeles, CA 90024

\section{R. A. Beaumont}

University of Washington

Seattle, WA 98105

C. C. Moore

University of California

Berkeley, CA 94720
J. DugunduI

Department of Mathematics

University of Southern California

Los Angeles, CA 90007

R. Finn AND J. Milgram

Stanford University

Stanford, CA 94305

\section{ASSOCIATE EDITORS}
E. F. BECKENBACH
B. H. NeumanN
F. WOLF
K. YoshidA

\section{SUPPORTING INSTITUTIONS}

UNIVERSITY OF BRITISH COLUMBIA

CALIFORNIA INSTITUTE OF TECHNOLOGY

UNIVERSITY OF CALIFORNIA

MONTANA STATE UNIVERSITY

UNIVERSITY OF NEVADA

NEW MEXICO STATE UNIVERSITY

OREGON STATE UNIVERSITY

UNIVERSITY OF OREGON

OSAKA UNIVERSITY

\author{
UNIVERSITY OF SOUTHERN CALIFORNIA \\ STANFORD UNIVERSITY \\ UNIVERSITY OF HAWAII \\ UNIVERSITY OF TOKYO \\ UNIVERSITY OF UTAH \\ WASHINGTON STATE UNIVERSITY \\ UNIVERSITY OF WASHINGTON \\ AMERICAN MATHEMATICAL SOCIETY
}

The Supporting Institutions listed above contribute to the cost of publication of this Journal, but they are not owners or publishers and have no responsibility for its contents or policies.

Mathematical papers intended for publication in the Pacific Journal of Mathematics should be in typed form or offset-reproduced (not dittoed), double spaced with large margins. Underline Greek letters in red, German in green, and script in blue. The first paragraph or two must be capable of being used separately as a synopsis of the entire paper. Items of the bibliography should not be cited there unless absolutely necessary, in which case they must be identified by author and Journal, rather than by item number. Manuscripts, in duplicate, may be sent to any one of the four editors. Please classify according to the scheme of Math. Reviews, Index to Vol. 39. All other communications should be addressed to the managing editor, or Elaine Barth, University of California, Los Angeles, California, 90024.

100 reprints are provided free for each article, only if page charges have been substantially paid. Additional copies may be obtained at cost in multiples of 50 .

The Pacific Journal of Mathematics is issued monthly as of January 1966. Regular subscription rate: $\$ 72.00$ a year (6 Vols., 12 issues). Special rate: $\$ 36.00$ a year to individual members of supporting institutions.

Subscriptions, orders for back numbers, and changes of address should be sent to Pacific Journal of Mathematics, 103 Highland Boulevard, Berkeley, California, 94708.

PUBLISHED BY PACIFIC JOURNAL OF MATHEMATICS, A NON-PROFIT CORPORATION

Printed at Jerusalem Academic Press, POB 2390, Jerusalem, Israel. 


\section{Pacific Journal of Mathematics}

Vol. 68, No. 2

April, 1977

William Allen Adkins, Aldo Andreotti and John Vincent Leahy, An analogue of Oka's theorem for weakly normal complex spaces ........

Ann K. Boyle, M. G. Deshpande and Edmund H. Feller, On nonsingularly

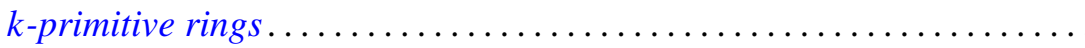

Rolando Basim Chuaqui, Measures invariant under a group of

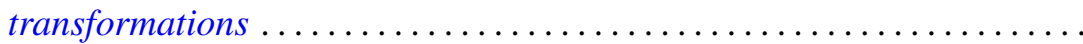

Wendell Dan Curtis and Forrest Miller, Gauge groups and classification of bundles with simple structural group .......................

Garret J. Etgen and Willie Taylor, The essential uniqueness of bounded nonoscillatory solutions of certain even order differential equations

Paul Ezust, On a representation theory for ideal systems

Richard Carl Gilbert, The deficiency index of a third order operator ........

John Norman Ginsburg, $S$-spaces in countably compact spaces using Ostaszewski's method.

Basil Gordon and S. P. Mohanty, On a theorem of Delaunay and some

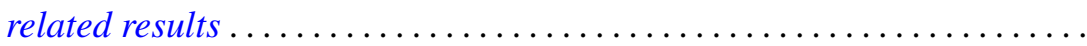

Douglas Lloyd Grant, Topological groups which satisfy an open mapping

theorem.

Charles Lemuel Hagopian, A characterization of solenoids

Kyong Taik Hahn, On completeness of the Bergman metric and its

subordinate metrics. II .

G. Hochschild and David Wheeler Wigner, Abstractly split group extensions.

Gary S. Itzkowitz, Inner invariant subspaces ...............

Jiang Luh and Mohan S. Putcha, A commutativity theorem for

non-associative algebras over a principal ideal domain.

Donald J. Newman and A. R. Reddy, Addendum to: "Rational approximation of $e^{-x}$ on the positive real axis".....

Akio Osada, On the distribution of a-points of a strongly annular function ....

Jeffrey Lynn Spielman, A characterization of the Gaussian distribution in a Hilbert space. .

Robert Moffatt Stephenson Jr., Symmetrizable-closed spaces ...

Peter George Trotter and Takayuki Tamura, Completely semisimple inverse $\Delta$-semigroups admitting principal series . . . . . . . .

Charles Irvin Vinsonhaler and William Jennings Wickless, Torsion free abelian groups quasi-projective over their endomorphism rings...

Frank Arvey Wattenberg, Topologies on the set of closed subsets ... 